\title{
New mutation of mitochondrial DNAJC19 causing dilated and noncompaction cardiomyopathy, anemia, ataxia, and male genital anomalies
}

\author{
Tiina Ojala' ${ }^{1}$ Padmini Polinati ${ }^{2}$, Tuula Manninen², Anita Hiippala', Jukka Rajantie ${ }^{3}$, Riitta Karikoski ${ }^{4}$, Anu Suomalainen ${ }^{2,5}$ \\ and Tiina Tyni ${ }^{2,6}$
}

BACKGROUND: We report a new mutation in the human DNAJC19 gene that causes early onset dilated cardiomyopathy syndrome (DCMA).

METHODS: Two brothers of Finnish origin presented with an unusual combination of early onset dilated cardiomyopathy syndrome, a disease which was associated with cardiac noncompaction, microcytic anemia, ataxia, male genital anomalies and methylglutaconic aciduria type V. Suspicion of a DCMA syndrome prompted sequencing of the human DNAJC19 gene.

RESULTS: Sequencing of the human DNAJC19 gene showed a homozygous single nucleotide (A) deletion in alanine 63 coding triplet in exon 6 , which does not immediately cause amino acid change but leads 11 amino acids later to a stop codon and to premature termination of the peptide. This DNAJC19 protein is located in the inner mitochondrial membrane and has been shown to function as a mitochondrial chaperone.

CONCLUSION: This is the first clinical report of DCMA syndrome, a human DNAJC19 deficiency, that is related to cases of severe dilated cardiomyopathy diagnosed in Europe. DNAJC19 deficiency causes a relatively specific finding in urinary organic acid analysis (methylglutaconic aciduria type V), which together with the clinical features of the ensuing cardiac disease, allows for effective screening before undertaking molecular genetic analysis.

C ardiomyopathies are a heterogeneous group of diseases with several etiologies ranging from heart specific muscle diseases (harboring defects in myosin, troponin, and other sarcomeric proteins) to generalized inherited mitochondrial disorders $(1,2)$. Developing a more complete understanding of the multitude of genetic causes and clinical features of these diseases is a major challenge.

An unusual etiology for early onset dilated cardiomyopathy is the autosomal and recessively inherited dilated cardiomyopathic syndrome (DCMA syndrome), which was characterized in 2006 in the Canadian Dariusleut Hutterite population
(3). This disorder is caused by a defect in the human mitochondrial protein DNAJC19 (MIM ID *608977), which is located in the inner mitochondrial membrane and has been shown to function as a mitochondrial chaperone. The reported DNAJC19 mutation from the Hutterite population involves a $\mathrm{G}$ to $\mathrm{C}$ transversion in the conserved AG splice acceptor site of intron 3 of the gene (IVS3-1GRC).

In addition to cardiomyopathy, DNAJC19 deficiency is also associated with several other specific clinical manifestations including growth failure, microcytic anemia, ataxia and male genital anomalies. Together with the clinical features of cardiomyopathic syndrome $(3,4)$, the ability to specifically detect this disorder by urinary organic acid analysis (3-methylglutaconic aciduria type $\mathrm{V}$ ) allows for robust screening of this disease before undertaking molecular genetic analysis (5). The DNAJC19 deficiency also has some similarities with X-linked Barth syndrome which is caused by a genetic defect in tafazzin, a mitochondrial transacylase required for cardiolipin remodeling. Barth syndrome is characterized by cardioskeletal myopathy, neutropenia and typical 3-methylglutaconic aciduria type II. The constitutive differences in the clinical presentations of these syndromes are the lack of skeletal myopathy and neutropenia in patients with a DNAJC19 deficiency. This study represents the first report of a DCMA syndrome outside of the Canadian Dariusleut Hutterite population. The clinical features of these patients expand the clinical presentation of DNAJC19 deficiency to include a noncompaction cardiomyopathy which has not been described earlier.

\section{RESULTS}

Molecular Genetic and Protein Expression Analyses

Genomic DNA was extracted from peripheral blood leukocytes using a standard phenol chloroform method as reported earlier (3). After detecting the mutation, an additional primer pair was designed for sequencing (forward primer 5'-GCTAAATCTCCCTCAGATAAG-3' and reverse primer 5' GAAGTTTAGACGGTAGGTAGTATAA-3'). Sequencing of

\footnotetext{
'Department of Pediatric Cardiology, University of Helsinki and Helsinki University Central Hospital, Helsinki, Finland; ${ }^{2}$ Research Program Unit, Molecular Neurology, Biomedicum Helsinki, University of Helsinki, Helsinki, Finland; ${ }^{3}$ Department of Pediatrics, University of Helsinki and Helsinki University Central Hospital, Helsinki, Finland; ${ }^{4}$ Department of Pathology, Helsinki University Central Hospital, Helsinki, Finland; ${ }^{5}$ Department of Neurology, University of Helsinki and Helsinki University Central Hospital, Helsinki, Finland; ${ }^{6}$ Department of Pediatric Neurology, Children's Hospital, University of Helsinki and Helsinki University Central Hospital, Helsinki, Finland.

Correspondence: Tiina Ojala (tiina.h.ojala@hus.fi)
} 
ATG GCC AGT ACA GTG GTA GCA GTT GGA CTG ACC ATT GCT GCT GCA GGA TTT GCA GGC CGT

$\begin{array}{llllllllllllllllllllll}M & A & S & T & V & V & A & V & G & \text { L } & \text { T } & \text { I } & \text { A } & \text { A } & \text { A } & \text { G } & F & A & G & R\end{array}$

TAC GTT TTG CAA GCC ATG AAG CAT ATG GAG CCT CAA CTA AAA CAA GTT TTT CAA AGC CTA

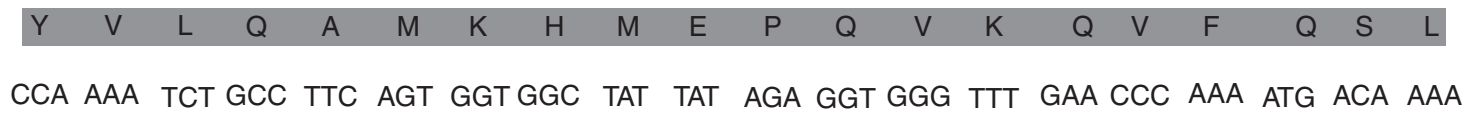

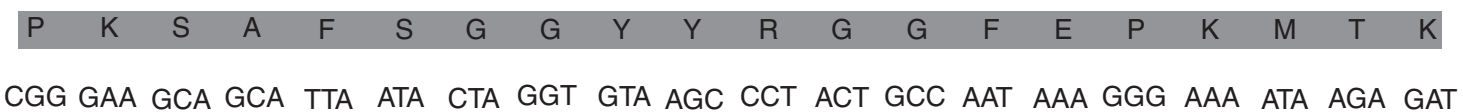

$\begin{array}{lllllllllllllllllllll}R & E & A & A & L & \text { I } & \text { L } & G & V & S & P & T & A & N & K & G & K & \text { I } & R & \text { D }\end{array}$
GCT CAT CGA CGA ATT ATG CTT TTA AAT CAT CCT GAC AAA GGA GGA TCT CCT TAT ATA GCA

$\begin{array}{cccccccccccccccccccc}\text { A } & H & \mathrm{R} & \mathrm{R} & \mathrm{I} & \mathrm{M} & \mathrm{L} & \mathrm{L} & \mathrm{N} & \mathrm{H} & \mathrm{P} & \mathrm{D} & \mathrm{K} & \mathrm{G} & \mathrm{G} & \mathrm{S} & \mathrm{P} & \mathrm{Y} & \text { I } & \text { A } \\ \text { GCC } & \text { AAA } & \text { ATC } & \text { AAT } & \text { GAA } & \text { GCT } & \text { AAA } & \text { GAT } & \text { TTA } & \text { CTA } & \text { GAA } & \text { GGT } & \text { CAA } & \text { GCT } & \text { AAA } & \text { AAA } & \text { TGA } & & & \end{array}$

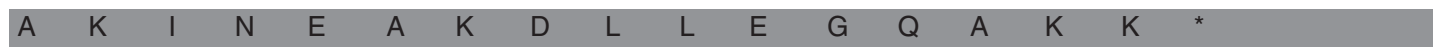

Figure 1. Human DNAJC19 gene sequencing revealed that both brothers harbored a homozygous single nucleotide (A) deletion c.300delA (p.Ala100fsX11) (http://www.ncbi.nlm.nih.gov/nuccore/NM_145261.3) within exon 6, which does not immediately cause an amino acid change but produces a stop codon 11 amino acids downstream and thus premature termination of the peptide. Filled box indicates a single base deletion; open box indicates single nucleotide polymorphism (rs17850540).

the human DNAJC19 gene showed that both brothers harbored a homozygous single nucleotide (A) deletion c.300delA (p.Ala100fsX11) (http://www.ncbi.nlm.nih.gov/nuccore/NM_ 145261.3) in exon 6, which does not immediately cause an amino acid change but produces a stop codon 11 amino acids later and thus premature termination of the peptide. In addition, the patients had a homozygous polymorphism c. $285 \mathrm{~A} \rightarrow \mathrm{C}$ (p.Gly95=) (rs17850540) (http://www.ncbi.nlm.nih.gov/SNP/ snp_ref.cgi?rs=17850540). Both parents had the same heterozygous single base deletion and polymorphism that was detected in the patients (Figure 1). Western blotting analysis was performed of the proteins extracted from cultured fibroblasts of the patients and from control cell lines (3). No DNAJC19 protein expression was detected in the patient sample, whereas a robust $10 \mathrm{kDa}$ band was detected in the control sample (Figure 2). The novel deletion mutation was not detected in a cohort of 22 children who had been diagnosed with a dilated or noncompaction cardiomyopathy.

\section{Patient 1}

Patient 1 was the first child of healthy, unrelated parents of Finnish origin. He was born at term after an uncomplicated pregnancy with a normal birth weight $(2770 \mathrm{~g},-2 \mathrm{SD})$ and height $(47 \mathrm{~cm},-2 \mathrm{SD})$. During infancy, his growth decreased from -2 to $-2.7 \mathrm{SD}$, weight increased from 0 to $15 \%$, and his head circumference decreased from 0 to -1 SD curve.

Laboratory findings. This patient had developed neonatal hypoglycemia. At 17 months, a microcytic, hypochromic anemia with slight anisocytosis was noted (hemoglobin $99 \mathrm{~g} / \mathrm{l}$,

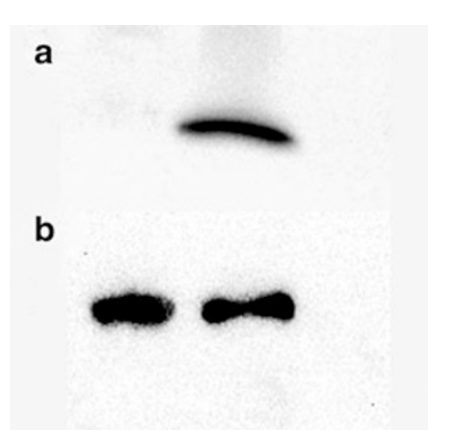

Figure 2. Western blotting analysis of the protein extracted from cultured fibroblasts of the patient and control cell lines: (a) DNAJC19 antibody and (b) a-actin antibody. No DNAJC19 protein expression was detected in the patient sample, whereas a robust band $(10 \mathrm{KDa})$ was found in the control sample.

erythrocyte count $4.98 \times 10 \mathrm{E} 12 / 1$ with $1.1 \%$ reticulocytes, mean red cell volume $64 \mathrm{fl}$, mean red cell hemoglobin $20 \mathrm{pg}$ and red cell distribution width $17 \%)$. The plasma ferritin concentration was $7 \mu \mathrm{g} / \mathrm{l}$ (the normal range for this age is $6-60 \mathrm{~g} / \mathrm{l}$ ) and the transferrin receptor concentration was $3.0 \mathrm{mg} / \mathrm{l}$ (normal, $1.5-3.3 \mathrm{mg} / \mathrm{l})$. Oral iron medication was administered and temporarily increased the hemoglobin and ferritin concentrations. However, the mean red cell volume remained subnormal and the red cell distribution was still higher than normal following this treatment.

The plasma alanine aminotransferase level was slightly elevated $(88 \mathrm{U} / \mathrm{l}$, normal $<40 \mathrm{U} / \mathrm{l})$ whereas the plasma lactate and lactate-pyruvate ratios were normal. Urinary organic acid analyses revealed a slightly elevated lactate level $(38 \mathrm{~mol} / \mathrm{mol}$ 


\section{Articles | ojala etal.}

creatinine, normal $<20$ ), a high excretion of methylglutaconic acid $(130 \mathrm{mmol} / \mathrm{l}$ creatinine, normal $<15)$ and mild increase in the excretion of 3-methylglutarate $(14 \mathrm{mmol} / \mathrm{mol}$ creatinine, normal $<5)$ and 2-ethylhydracrylate $(37 \mathrm{mmol} / \mathrm{mol}$ creatinine, normal $<20)$. The serum desialotransferrin levels were slightly elevated $(2.2 \%$, normal $<1.8 \%)$, but showed a normal profile.

Neurological and respiratory chain findings. The motor development in patient 1 was mildly delayed: he learned to walk unsupported at 1 year and 7 months, produced single words at 1 year and sentences at 2 years. He also had problems with balance, and showed a mild ataxia, gradual deterioration of fine motor skills, mild muscle weakness and a slightly reduced muscular mass. Brain magnetic resonance imaging results were normal at 3 years of age. A quadriceps femoris muscle biopsy showed a mild neutral lipid accumulation in single muscle fibers, but histochemical activities of cytochrome $\mathrm{C}$ oxidase and succinate dehydrogenase were normal. Biochemical analysis of mitochondrial function showed a slightly reduced adenosine triphosphate production with most substrates, and mitochondrial complex CI, CII, and CIV dependent enzyme activities were partially reduced suggesting partial respiratory chain enzyme deficiency in skeletal muscle.

Cardiac findings. At 2 years of age, a systolic murmur was audible and cardiac examinations were therefore introduced. A small muscular ventricular septal defect was subsequently found. Cardiac ultrasound analysis further demonstrated an enlarged left ventricle with an end diastolic diameter of $36 \mathrm{~mm}(+3.5 \mathrm{SD})$ and a mild decrease in systolic and diastolic function (fractional shortening, $27 \%$ ). The left ventricular posterior wall and infero-apical myocardium were mildly noncompacted. A maximum end systolic ratio of the noncompacted endocardial layer to the compacted myocardium of $3.0 \mathrm{~mm} / 7.0 \mathrm{~mm}$ was characterized. Color Doppler echocardiograms revealed perfused intertrabecular recesses. Electrocardiography further showed a prolonged QT interval corrected for heart rate (QTc $520 \mathrm{~ms})$. Cardiac catheterization was performed and an aortic root angiography showed a normal coronary anatomy. The left ventricular end diastolic pressure measured 9-11 $\mathrm{mm} \mathrm{Hg}$ and $\alpha$ - and $\beta$-adrenergic blocking medication (carvedilol $0.5 \mathrm{mg} / \mathrm{kg} / \mathrm{day}$ ) was then initiated. After 6 months, the cardiac systolic and diastolic function had normalized (fractional shortening $>30 \%$, the ratio of transmitral Doppler early filling velocity to tissue Doppler early diastolic mitral annular velocity $\left.\left(\mathrm{E} / \mathrm{E}^{\prime}\right)\right)(6)$. The left ventricular end diastolic diameter was still mildly enlarged $(+2.5 \mathrm{SD})$. Because of the prolonged QTc-interval, carvedilol was replaced with propranolol ( $3 \mathrm{mg} / \mathrm{kg} /$ day). Repeat 24 -h Holter registrations did not reveal any rhythm disturbances. Ubiquinone $(9 \mathrm{mg} / \mathrm{kg} /$ day $)$ and carnitine $(9 \mathrm{mg} / \mathrm{kg} /$ day $)$ were then administered as a combination treatment. The overall clinical condition of this patient remained relatively stable thereafter and at present he has no clinical cardiac symptoms (Figure 3 ).

\section{Patient 2}

Patient 2 was the second child of the same family as patient 1. He was born at 38 gestational weeks with normal birth metrics: weight, $2825 \mathrm{~g}(-1 \mathrm{SD})$; height, $48 \mathrm{~cm}(-1.5 \mathrm{SD})$; and head circumference, $34 \mathrm{~cm}(-1 \mathrm{SD})$. Later in infancy however, this patient showed progressive growth retardation (height -3.7 $\mathrm{SD}$, head growth $-2.2 \mathrm{SD}$ ) and also a right sided cryptorchism and chorda penis.

Laboratory findings. Neonatally, this patient had mild hypoglycemia, as found in his older brother, patient 1 . He also had constant microcytic anemia. At 10 months of age, his hemoglobin was $93 \mathrm{~g} / \mathrm{l}$, and he had an erythrocyte count of $4.01 \times$ $10 \mathrm{E} 12 / 1$ with $1.1 \%$ reticulocytes, a mean red cell volume 71 $\mathrm{fl}$, a mean red cell hemoglobin $23 \mathrm{pg}$, and a red cell distribution width of $17 \%$. He received no iron therapy. The plasma alanine aminotransferase, plasma lactate and lactate-pyruvate ratios were normal. The findings of urinary organic acid analysis were also normal for this patient at 10 months. His serum desialotransferrin levels were slightly elevated (2.2\%, normal $<1.8 \%$ ), but showed normal profiles.

Neurological findings. Patient 2 showed a moderate muscular hypotonia. Brain ultrasound imaging results were normal.

Cardiac findings. Fetal echocardiography was performed at 20 weeks of gestation due to the cardiac history of the older brother and normal cardiac findings were obtained. Three months postnatally however, echocardiography revealed that the ventricular free wall and apical part of the myocardium were noncompacted (7). The maximum end systolic ratio of the noncompacted endocardial layer to the compacted myocardium was $9.0 \mathrm{~mm} / 5.0 \mathrm{~mm}$. The left ventricular end diastolic diameter was normal at $25 \mathrm{~mm}(+1.5 \mathrm{SD})$. Cardiac systolic-diastolic function was mildly compromised (fractional shortening $26 \%, \mathrm{E} / \mathrm{E}^{\prime} 14$ ) and the QTc-interval ranged from 330 to $470 \mathrm{~ms}$, with no arrhythmia detected in a $24-\mathrm{h}$ Holter registration. Carvedilol $(0.5 \mathrm{mg} / \mathrm{kg} /$ day) was administrated to treat the mild cardiac dysfunction but the left ventricle end diastolic diameter still increased to $37 \mathrm{~mm}$ (+4 SD). Cardiac function was then found to be substantially compromised by the age of 10 months and enalapril $(0.5 \mathrm{mg} /$ $\mathrm{kg} /$ day $)$, spironolactone $(2 \mathrm{mg} / \mathrm{kg} /$ day $)$, and warfarin were administered as a combination therapy. One month later, oral ubiquinone $(9 \mathrm{mg} / \mathrm{kg} /$ day $)$ and carnitine $(9 \mathrm{mg} / \mathrm{kg} /$ day $)$ were administered. The patient subsequently developed symptoms of heart failure (vomiting and tachypnea) and his condition deteriorated rapidly leading to a short loss of consciousness and cardiac decompensation. A milrinone infusion was initiated but by 13 months of age the patient had progressive heart failure. Following discussion with the parents, intensive therapeutic measures and resuscitation were withheld (Figure 3 ).

Autopsy findings. A neuropathological macroscopic examination showed that the structures of the brain stem were mildly atrophied, which could explain the ataxia of the patients. The pons, medulla oblongata and cerebellum were normal. Microscopic analysis revealed a minor diffuse atrophy in the brain stem and a minor loss of neurons and vacuolization in the periaqueductal neuropil. Scant vacuolization was found in the pontine 

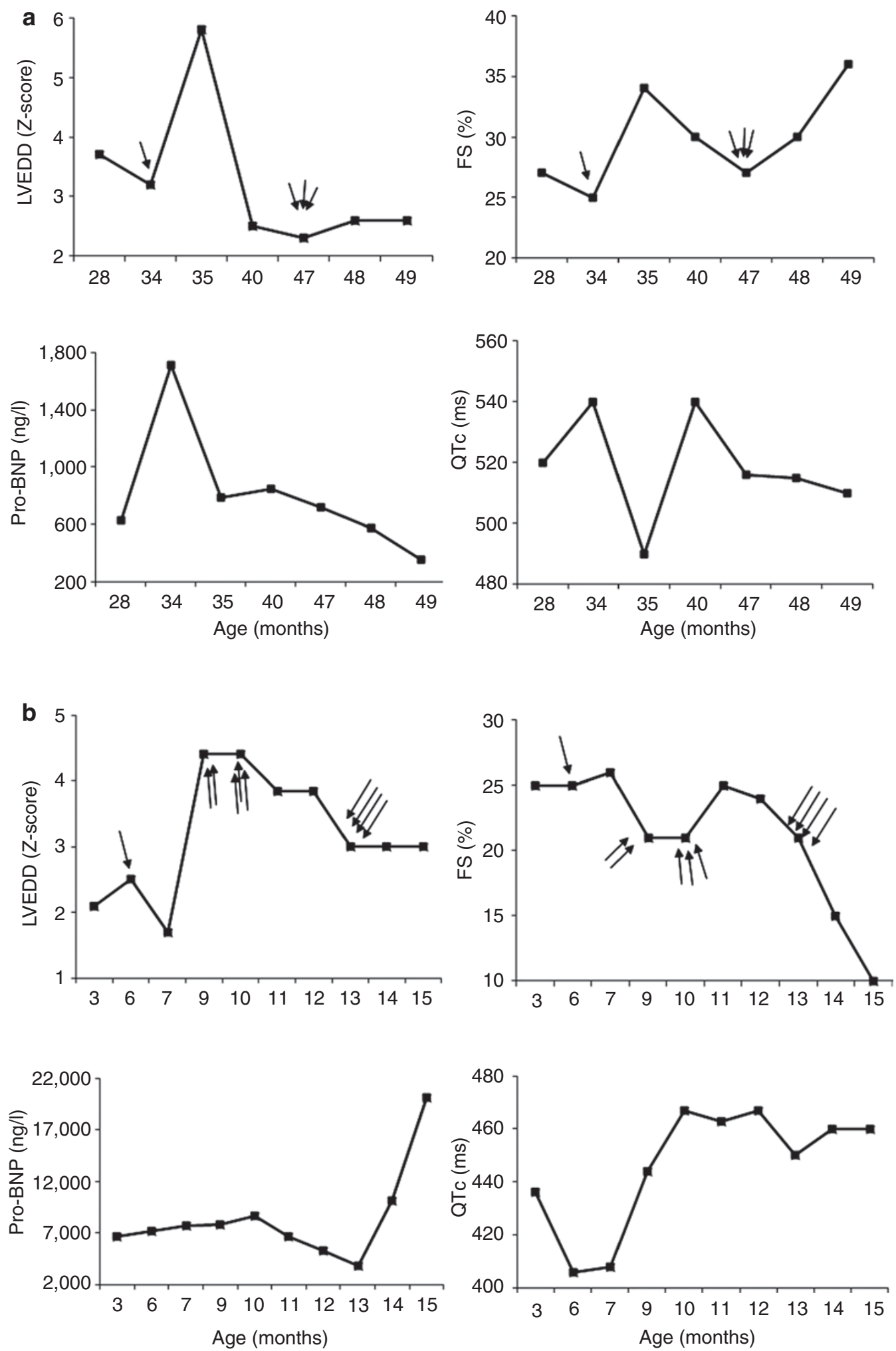

Figure 3. Follow-up analyses of the left ventricular end diastolic dimension Z-scores, fractional shortening (FS), serum levels of N-terminal probrain natriuretic peptide (proBNP), and QTc time intervals in the (a) older and (b) younger siblings. The cardiac function of patient 1 improved and mild left ventricular remodeling was observed following treatment for heart failure. In contrast, there were no significant improvements in the cardiac function of patient 2 following various treatments. Nutritional supplementation of carnitine (responsible for the transport of fatty acids into the mitochondria) and ubiquinone (coenzyme Q10, a component of the electron transport chain) was initiated to improve heart function (two arrows) but no response was detected. Treatments included the administration of a $\beta$-blocker (single arrow); enalapril, spironolactone, and warfarin therapy (two arrows); ubiquinone and carnitine (three arrows); intravenous milrinone (four arrows). 


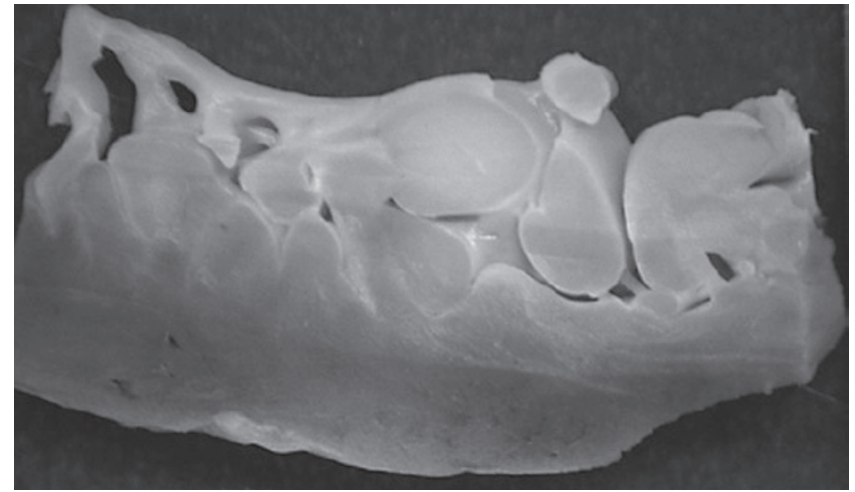

Figure 4. Transmural, morphologic section of the apical portion of the left ventricle. Both an epicardial compacted layer and an endocardial noncompacted layer with prominent trabeculations and deep recesses are visible.

tegmentum. The cerebellar structures were intact but the neuronal density of the dentate nucleus was marginally reduced and pycnotic cell and minor fiber depletion was evident in the hilus.

A cardiac examination confirmed that the left ventricle was dilated and noncompacted (Figure 4). The left ventricle free wall and apical myocardium showed a two-layered structure including a thin, compacted epicardial band and a much thicker, noncompacted endocardial layer consisting of a trabecular meshwork with endocardial spaces. A ratio of the noncompacted endocardial layer to the compacted myocardium was $9 \mathrm{~mm} / 5 \mathrm{~mm}$. Blue native gel analysis of the respiratory chain complexes showed normal amounts of mitochondrial respiratory chain enzymes in the skeletal and heart muscles (Figure 5).

\section{DISCUSSION}

To our knowledge, this is the first report of a DNAJC19 gene defect causing an early onset cardiomyopathy syndrome, DCMA syndrome, outside of the Canadian Dariusleut Hutterite population. Clinical features of these patients extend the cardiac features of DNAJC19 disease from a purely dilated cardiomyopathy to a shared dilated and noncompaction cardiomyopathy. Other clinical features of this defect were found to be similar to those earlier reported for the Canadian Hutterite population $(3,4)$. In the largest yet published cohort of over 30 patients with DNAJC19 deficiency, the mean onset of disease was 12 months (range 1-36 months) (4). All of the affected patients had growth failure, and the other symptom frequencies among the cohort were as follows: $67 \%$ anemia, $61 \%$ dilated cardiomyopathy, $55 \%$ mild or borderline mental retardation, $55 \%$ ataxia, 33\% long QT and 22\% optic atrophy. In addition, most of the affected males had genital abnormalities including cryptorchism and hypospadia. Long QT syndrome was detected both with and without cardiomyopathy. Congestive heart failure and/or arrhythmias led to early death (mean 22 months, range $4-48$ months) in $60 \%$ of these patients $(3,4)$.

Mutations of the X-linked tafazzin gene cause Barth syndrome, which shares several features in common with DNAJC19 deficiency including early onset cardiomyopathy, methylglutaconic aciduria and growth failure (8). Cerebellar

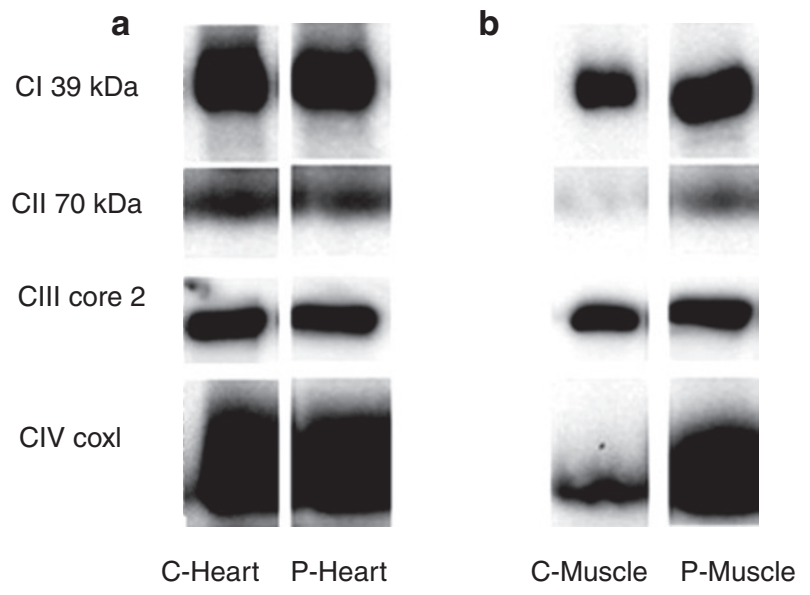

Figure 5. Blue native electrophoretic analyses of mitochondrial respiratory chain complexes in the (a) heart and (b) skeletal muscle of patient 1 $(\mathrm{P})$ in comparison with control samples (C). Ten micrograms of each protein sample was loaded onto the gel. For protein detection, monoclonal antibodies (Mitosciences, Eugene, OR) against the $39 \mathrm{kDa}$ subunit of Complex I (CI), the core 2 or Rieske subunits of Complex III (CIII), the cox $1 \mathrm{p}$ subunits of Complex IV (CIV), and the $70 \mathrm{kDa}$ Ip subunit of Complex II (CII) were used. This analysis revealed no abnormalities in the mitochondrial respiratory chain complexes in the heart or skeletal muscle of patient 1.

symptoms and autosomal recessive inheritance are characteristic of a DNAJC19 deficiency but are not seen in Barth syndrome. Both DNAJC19 and tafazzin are mitochondrial proteins whereas DNAJC19 functions as a mitochondrial chaperone. A tafazzin knockdown results in a cardiolipin deficiency, reduced mitochondrial adenosine triphosphate production, decreased cardiac contractility, hypertrophy and cell death (6). Overall, little is currently known about the inherited genetic defects affecting human DNAJ proteins and other mitochondrial chaperones (9). Although DNAJC19 and tafazzin defects cause early onset cardiomyopathies, genetic defects in BCL2 (B-cell lymphoma 2) -associated athanogene 3 (BAG3), a co-chaperone of the heat shock proteins, have recently been shown to cause adult onset dilated cardiomyopathy $(3,10)$.

In contrast to patients with Barth syndrome, neither cardiac noncompaction nor hypertrophy have been previously detected in patients with a DNAJC19 deficiency. Noncompaction of the ventricular myocardium is a recently recognized congenital cardiomyopathy, characterized by a spongy appearance of the ventricular myocardium with deep intertrabecular recesses predominantly involving the apical and posterior portion of the left ventricle $(11,12)$. Left ventricular noncompaction has been suggested to result from an arrest during normal embryogenesis (13). In this study, however, the echocardiogram of the younger brother appeared to be normal at 20 weeks of gestation. It is not clear whether these findings were related to limitations of the fetal echocardiography technique or to the postnatal development of the disorder (14). Left ventricular noncompaction has mostly been associated with disorders causing skeletal muscle or cardiac disease, or with chromosomal anomalies $(15,16)$. 
Both isolated and familial cases have been reported (17). The combination of cardiomyopathy phenotypes (dilated and noncompacted) observed in our patients support the recent suggestions that there are shared molecular etiologies for different types of cardiomyopathies (18).

Although it is often difficult to obtain a specific etiological diagnosis in cases of pediatric cardiomyopathy, in the future it will be even more important to differentiate systemic metabolic diseases from disorders that affect only the cardiac muscle. Understanding the biochemical and genetic features of cardiomyopathies will enable us to comprehend their clinical heterogeneity. Clinical features of our patients show that a DNAJC19 deficiency may manifest as both a dilated and left ventricular noncompaction cardiomyopathy. Detection of noncompaction may be an important factor in assessing disease severity as noncompaction may essentially increase the risk of poor ventricular function, arrhythmias and thrombosis (12).

In conclusion, this is the first clinical report of DCMA syndrome, a human DNAJC19 deficiency, that is related to cases of severe dilated cardiomyopathy diagnosed in Europe. DNAJC19 deficiency causes a relatively specific finding in urinary organic acid analysis (methylglutaconic aciduria type $\mathrm{V}$ ), which together with the clinical features of the ensuing cardiac disease, allows for effective screening before undertaking molecular genetic analysis.

\section{PATIENTS AND METHODS}

Two brothers of Finnish origin were presented with an unusual combination of a cardiomyopathy with growth failure, microcytic anemia, ataxia, neonatal hypoglycemia, mild liver involvement, muscle hypotonia, cryptorchism and chorda penis. The parents were healthy and reported to be unrelated to each other. Since the clinical features of these siblings were suggestive of either Barth syndrome or DNAJC19 deficiency, blood and urine samples were collected from the older brother for biochemical analyses. These included assays for plasma alanine aminotransferase, desialotransferrin, lactate and pyruvate levels, in addition to urinary organic acid analysis. A skeletal muscle biopsy was also performed. The detection of mild lactic acidemia and methylglutaconic aciduria as well as the lack of skeletal myopathy and neutropenia in the older brother strengthened the suspicion of a DCMA syndrome, and prompted sequencing of the human DNAJC19 gene (3). This study is part of a larger project aimed at improving the etiologic diagnostics available for Finnish children with cardiomyopathy. Written informed consent was obtained from the patients' parents and the study protocols were approved by the Ethical committee of Helsinki University Central Hospital, Helsinki, Finland.

\section{ACKNOWLEDGMENTS}

The authors appreciate the assistance of Alexandra Götz in blue native electrophoretic analyses of mitochondrial respiratory chain complexes.

\section{STATEMENT OF FINANCIAL SUPPORT}

This work was supported by grants from the Finnish Pediatric Research Foundation, Finnish Medical Foundation, Academy of Finland, Jane and Aatos Erkko Foundation, Sigrid Jusélius Foundation, and Helsinki University.

\section{REFERENCES}

1. Elliott $\mathrm{P}$, Andersson B, Arbustini E, et al. Classification of the cardiomyopathies: a position statement from the European Society Of Cardiology Working Group on Myocardial and Pericardial Diseases. Eur Heart J 2008; 29:270-6.

2. Maron BJ, Towbin JA, Thiene G, et al. Contemporary definitions and classification of the cardiomyopathies: an American Heart Association Scientific Statement from the Council on Clinical Cardiology, Heart Failure and Transplantation Committee; Quality of Care and Outcomes Research and Functional Genomics and Translational Biology Interdisciplinary Working Groups; and Council on Epidemiology and Prevention. Circulation 2006;113:1807-16.

3. Davey KM, Parboosingh JS, McLeod DR, et al. Mutation of DNAJC19, a human homologue of yeast inner mitochondrial membrane co-chaperones, causes DCMA syndrome, a novel autosomal recessive Barth syndrome-like condition. J Med Genet 2006;43:385-93.

4. Sparkes R, Patton D, Bernier F. Cardiac features of a novel autosomal recessive dilated cardiomyopathic syndrome due to defective importation of mitochondrial protein. Cardiol Young 2007;17:215-7.

5. Wortmann SB, Kluijtmans LA, Engelke UF, Wevers RA, Morava E. The 3-methylglutaconic acidurias: what's new? J Inherit Metab Dis 2012; 35:13-22.

6. He Q. Tafazzin knockdown causes hypertrophy of neonatal ventricular myocytes. Am J Physiol Heart Circ Physiol 2010;299:H210-6.

7. Jenni R, Oechslin E, Schneider J, Attenhofer Jost C, Kaufmann PA. Echocardiographic and pathoanatomical characteristics of isolated left ventricular non-compaction: a step towards classification as a distinct cardiomyopathy. Heart 2001;86:666-71.

8. Bione S, D'Adamo P, Maestrini E, Gedeon AK, Bolhuis PA, Toniolo D. A novel X-linked gene, G4.5. is responsible for Barth syndrome. Nat Genet 1996;12:385-9.

9. Kampinga HH, Craig EA. The HSP70 chaperone machinery: J proteins as drivers of functional specificity. Nat Rev Mol Cell Biol 2010;11:579-92.

10. Norton N, Li D, Rieder MJ, et al. Genome-wide studies of copy number variation and exome sequencing identify rare variants in BAG3 as a cause of dilated cardiomyopathy. Am J Hum Genet 2011;88:273-82.

11. Ichida F, Hamamichi Y, Miyawaki T, et al. Clinical features of isolated noncompaction of the ventricular myocardium: long-term clinical course, hemodynamic properties, and genetic background. J Am Coll Cardiol 1999;34:233-40.

12. Ergul Y, Nisli K, Demirel A, et al. Left ventricular non-compaction in children and adolescents: clinical features, treatment and follow-up. Cardiol J 2011;18:176-84.

13. Jenni R, Oechslin EN, van der Loo B. Isolated ventricular non-compaction of the myocardium in adults. Heart 2007;93:11-5.

14. Bleyl SB, Mumford BR, Brown-Harrison MC, et al. Xq28-linked noncompaction of the left ventricular myocardium: prenatal diagnosis and pathologic analysis of affected individuals. Am J Med Genet 1997;72:257-65.

15. Arunamata A, Punn R, Cuneo B, Bharati S, Silverman NH. Echocardiographic diagnosis and prognosis of fetal left ventricular noncompaction. J Am Soc Echocardiogr 2012;25:112-20.

16. Pignatelli RH, McMahon CJ, Dreyer WJ, et al. Clinical characterization of left ventricular noncompaction in children: a relatively common form of cardiomyopathy. Circulation 2003;108:2672-8.

17. Finsterer J. Cardiogenetics, neurogenetics, and pathogenetics of left ventricular hypertrabeculation/noncompaction. Pediatr Cardiol 2009;30: 659-81.

18. Arad M, Penas-Lado M, Monserrat L, et al. Gene mutations in apical hypertrophic cardiomyopathy. Circulation 2005;112:2805-11. 